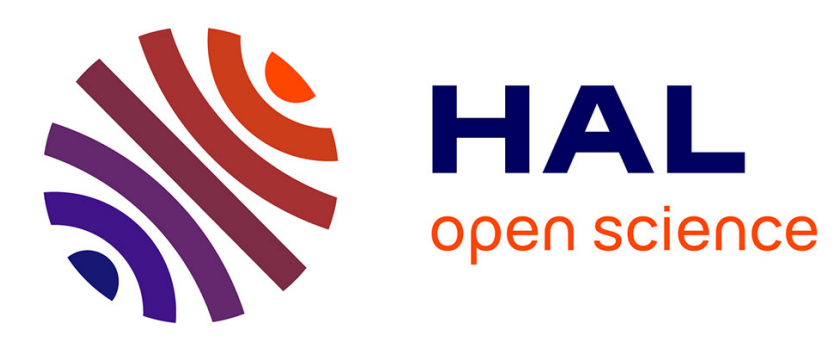

\title{
Exact bounds on the effective behaviour of a conducting discrete polycrystal
}

\author{
Andrea Braides, Antoine Gloria
}

\section{To cite this version:}

Andrea Braides, Antoine Gloria. Exact bounds on the effective behaviour of a conducting discrete polycrystal. Multiscale Modeling and Simulation: A SIAM Interdisciplinary Journal, 2008, 6 (4), pp.1198-1216. 10.1137/06067184X . hal-00766730

\section{HAL Id: hal-00766730 \\ https://hal.inria.fr/hal-00766730}

Submitted on 19 Dec 2012

HAL is a multi-disciplinary open access archive for the deposit and dissemination of scientific research documents, whether they are published or not. The documents may come from teaching and research institutions in France or abroad, or from public or private research centers.
L'archive ouverte pluridisciplinaire HAL, est destinée au dépôt et à la diffusion de documents scientifiques de niveau recherche, publiés ou non, émanant des établissements d'enseignement et de recherche français ou étrangers, des laboratoires publics ou privés. 


\title{
EXACT BOUNDS ON THE EFFECTIVE BEHAVIOUR OF A CONDUCTING DISCRETE POLYCRYSTAL
}

\author{
ANDREA BRAIDES AND ANTOINE GLORIA *
}

\begin{abstract}
In a recent paper by Braides and Francfort, the problem of the characterization of the overall properties of lattice energies describing networks with arbitrary mixtures of two types of linear conductors has been addressed in a two-dimensional setting. In this paper we investigate the connection between that discrete optimization process and the theory of bounds for mixtures of continuum energies, for which the choice of the relationships between the different phases of the mixture is unusual and leads to remarkably simple results in terms of $G$-closure.
\end{abstract}

Key words. polycrystals, discrete energies, effective properties, composite materials.

AMS subject classifications. 77Q15, 74S05, 35J60

1. Introduction. In a paper by Braides and Francfort [6], the problem of the characterization of the overall properties of lattice energies describing networks with arbitrary mixtures of two types of linear conductors has been addressed. In a twodimensional setting, with the notation of $\Gamma$-convergence, that problem translates into the limit analysis as $h \rightarrow 0$ of discrete energies of the type

$$
E_{h}(u)=\frac{1}{2} \sum_{(i, j) \in \mathcal{N}_{h}} c_{i j}^{h}\left(u_{i}-u_{j}\right)^{2} \quad u: \mathcal{N}_{h} \rightarrow \mathbb{R},
$$

where $\mathcal{N}_{h}$ denotes the set of nearest neighbours (i.e., pairs $(i, j)$ such that $|i-j|=h$ ) on a portion of a square lattice $h \mathbb{Z}^{2} \cap \Omega, \Omega$ being a regular open subset of $\mathbb{R}^{2}$, and

$$
c_{i j}^{h} \in\{\alpha, \beta\}
$$

are chosen arbitrarily. It should be noted that in the extreme cases $c_{i j}^{h} \equiv \alpha$ and $c_{i j}^{h} \equiv \beta$ the functionals above are simply discretizations of the Dirichlet integral with the corresponding coefficient. Since the analogue of the 'localization principle' holds in this discrete setting, the $\Gamma$-limit problem translates in a sort of 'discrete $G$-closure problem', where bounds on a matrix given by a 'discrete homogenization formula' must be exhibited.

The results in [6] show that all possible limits of $E_{h}$ have the form

$$
F(u)=\int_{\Omega}\langle A(x) \nabla u, \nabla u\rangle d x \quad u \in H^{1}(\Omega),
$$

with $A(x)$ belonging to a set of matrices determined only by the local limit proportion $\theta(x)$ at $x$ of ' $\alpha$-connections'. Such sets can be compared with those obtained by limits of mixtures of isotropic energies; i.e., of functionals of the form

$$
E_{\epsilon}(u)=\int_{\Omega}\left(\alpha \chi_{\Omega_{\epsilon}}+\beta\left(1-\chi_{\Omega_{\epsilon}}\right)\right)|\nabla u|^{2} d x \quad u \in H^{1}(\Omega),
$$

\footnotetext{
*Dipartimento di Matematica, Università di Roma 'Tor Vergata',via della ricerca scientifica 1, 00133 Rome, Italy (braides@mat .uniroma2 . it ), CERMICS, Ecole Nationale des Ponts et Chaussées, 6 et 8 avenue Blaise Pascal, Cité Descartes, 77455 Marne-la-Vallée Cedex 2, FRANCE, and projet MICMAC, INRIA, Rocquencourt (gloria@cermics.enpc.fr)
} 


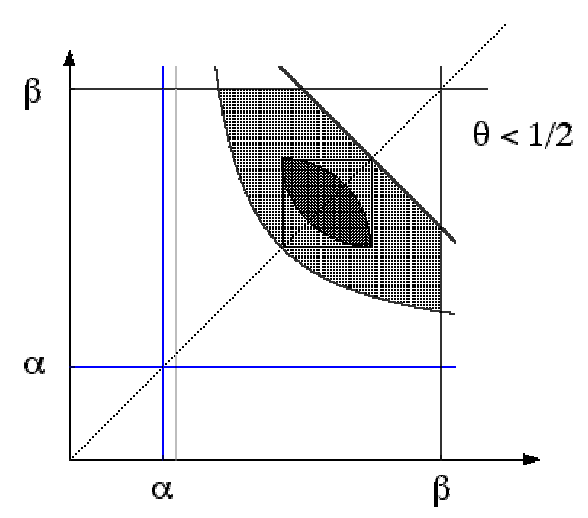

FIG. 1.1. Comparison of the bounds for discrete and isotropic continuous materials

with $\Omega_{\epsilon}$ arbitrary measurable subsets of $\Omega\left(\chi_{\Omega_{\epsilon}}\right.$ denotes the corresponding characteristic function). These limits have the same form $F$ with $A(x)$ now belonging to a set whose form depends on the limit volume fraction $\theta(x)$ of the $\alpha$-phase; i.e., on the value at $x$ of the weak ${ }^{*}$-limit of $\chi_{\Omega_{\epsilon}}$ (see Murat and Tartar $[12,13]$ and Cherkaev and Lurie [10]). In Figure 1.1 the two problems are compared in terms of the possible eigenvalues of $A(x)$ for some $\theta(x)<1 / 2$ : the shaded sets represent all possible pairs of eigenvalues in the two cases, the smaller set being the 'continuous' one (see Tartar $[16,17])$. This comparison shows that the discrete energies cannot be simply interpreted as a mixture of the two 'extremal' isotropic energies.

The discrete functionals can be heuristically interpreted as 'anisotropic' continuous ones in the following way, pictured in Figure 1.2. We introduce a triangulation
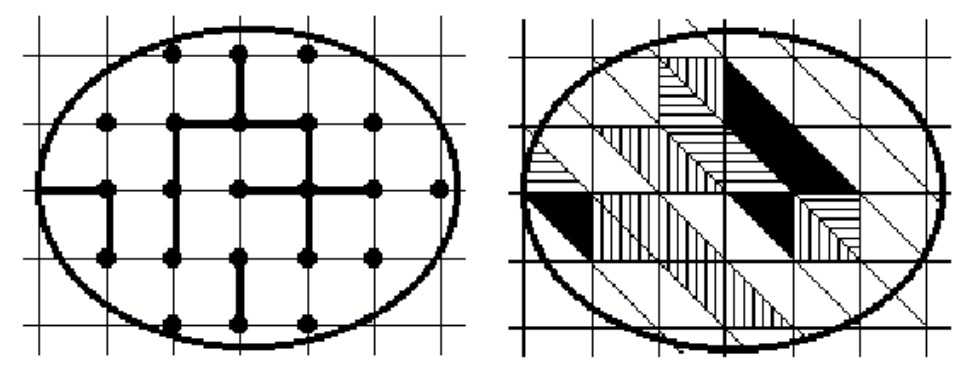

FIG. 1.2. a conducting network and the corresponding continuous coefficients

with underlying lattice $h \mathbb{Z}^{2}$, and to every triangle $T$ with a horizontal side corresponding to a connection with value $c_{i j}^{h}=c_{h}$ and a vertical side corresponding to a connection with $c_{i j}^{h}=c_{v}$ we associate the matrix

$$
\widetilde{A}_{h}(x)=\left(\begin{array}{cc}
c_{h} & 0 \\
0 & c_{v}
\end{array}\right) \quad \text { for } x \in T .
$$

In Figure 1.2, on the left, 'thick connections' correspond to the coefficient $\beta$ (the other ones to $\alpha$ ), while on the right on black triangles we take $\widetilde{A}_{h}(x)=\beta I$, on white triangles $\widetilde{A}_{h}(x)=\alpha I$, and on the remaining triangles the anisotropic conductivities. 
Note that $\widetilde{A}_{h}$ takes only four possible values. Chosing $\tilde{u}_{h} \in H^{1}(\Omega)$ as the piecewise affine interpolation of $u_{h}$ defined on $\mathcal{N}_{h}$, we have

$$
E_{h}\left(u_{h}\right)=\widetilde{E}_{h}\left(\tilde{u}_{h}\right)+o(1),
$$

(the last term deriving from an asymptotically negligible boundary term; see e.g. [1]) where

$$
\widetilde{E}_{h}(u)=\int_{\Omega}\left\langle\widetilde{A}_{h}(x) \nabla u, \nabla u\right\rangle d x \quad u \in H^{1}(\Omega) .
$$

Note that the infimum of $\widetilde{E}_{h}$ on $H^{1}(\Omega)$ is in general strictly lower than the corresponding one for $E_{h}$ provided compatible boundary conditions are given. On the other hand, any continuous configuration can be approximated by a discrete configuration (let simply think of the discretization of the Dirichlet integral). Therefore, the $\Gamma$-closure of the discrete energies $E_{h}$ contains the $\Gamma$-closure of the continuous energies $\widetilde{E}_{h}$. Whether this inclusion is strict or not is unclear. However, we will show that for polycristalline structures the two closures coincide.

Continuous energies as $\widetilde{E}_{h}$ can also be thought of as being a polycrystalline mixture of three conducting materials by regarding one anisotropic value of $\widetilde{A}_{h}$ as the rotation of the other one, so that we may rewrite

$$
\widetilde{E}_{h}(u)=\int_{\Omega}\left\langle A_{h}(x) R_{h}(x) \nabla u(x), R_{h}(x) \nabla u(x)\right\rangle d x, \quad u \in H^{1}(\Omega),
$$

with $A_{h}(x)$ taking one of the values

$$
\left(\begin{array}{ll}
\alpha & 0 \\
0 & \alpha
\end{array}\right),\left(\begin{array}{ll}
\beta & 0 \\
0 & \beta
\end{array}\right), \quad \text { or } \quad\left(\begin{array}{ll}
\alpha & 0 \\
0 & \beta
\end{array}\right)
$$

and the rotation matrix $R_{h}(x)$ being either the identity or the rotation by ninety degrees. A more natural setting for the continuous energy is obtained by removing the geometric restrictions due to the lattice symmetries, and determine all the possible conductivity tensors that can be obtained as possible $\Gamma$-limits of energies

$$
F_{\epsilon}(u)=\int_{\Omega}\left\langle A_{\epsilon}(x) R_{\epsilon}(x) \nabla u(x), R_{\epsilon}(x) \nabla u(x)\right\rangle d x,
$$

with $A_{\epsilon}$ and $R_{\epsilon}$ families of matrices, with $A_{\epsilon}$ taking the three values above, and $R_{\epsilon}$ arbitrary rotations. If one adds the restriction that $R_{\epsilon}$ be piecewise-constant, then we may interpret this continuous energy as a limit of discrete polycrystals, that is a polycrystal obtained by a discrete to continuum process. This discrete polycrystalline material is composed of grains of diameter much larger than the lattice spacing $h$, at whose interior we consider a network structure whose lattice orientation is described by the rotation matrix $R_{\epsilon}(x)$, as depicted in Figure 1.3 (the black and grey subdomains represent isotropic parts of the energy whereas the other subdomains correspond to underlying lattices oriented according to the arrows). In this setting, the characterization of the $\Gamma$-closure of such discrete polycrystals is complete since the additional invariance by rotation of the $\Gamma$-limit allows us to focus on diagonal matrices, which have been fully described in [5].

The scope of the present paper is twofold: to prove that the bounds obtained by considering piecewise-constants rotations $R_{\epsilon}$ still hold for general rotations, and 


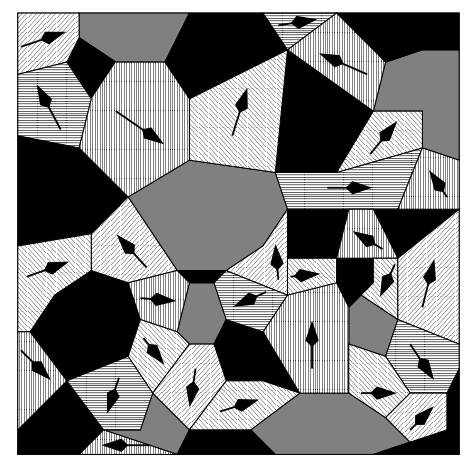

FIG. 1.3. Polycrystal

that these bounds - which are optimal in the discrete setting - are also attained in this continuous setting. This amounts to proving that the $\Gamma$-closures of discrete and continuous polycrystals are the same. In what follows, these two types of polycrystal will be refered to as 'discrete' since the $\Gamma$-closures are related to the very special relationship between the phases, that is inherited from the discrete description.

From a modeling viewpoint, these energies can equivalently be thought of as describing a bilayered polycrystal made of two types of layers (namely $\mathcal{A}$ and $\mathcal{B}$ ). Each layer has a conductivity tensor $C \nu \otimes \nu$, where $\nu \in S^{1}\left(\mathbb{R}^{2}\right), C=\alpha$ for type $\mathcal{A}$ and $C=\beta$ for type $\mathcal{B}$, with $\beta>\alpha>0$. Each simple crystal is a bilayer $C_{1} \nu_{1} \otimes \nu_{1}+C_{2} \nu_{2} \otimes \nu_{2}$ with $C_{i} \in\{\alpha, \beta\}$ obtained by the superposition of two simple layers in orthogonal directions $\nu_{1} \perp \nu_{2}$, such that the conductivity tensor of the crystal is of the form above (1.1). The association of rotated simple crystals gives rise to the polycrystal under investigation.

It is convenient to rewrite the energies $F_{\epsilon}$ in (1.2) in a different form, as

$$
\begin{aligned}
F_{\epsilon}(u)=\alpha \int_{E_{1}^{\epsilon}}|\nabla u|^{2} d x+\beta \int_{\Omega \backslash E_{2}^{\epsilon}}|\nabla u|^{2} d x & \\
& \quad+\int_{E_{2}^{\epsilon} \backslash E_{1}^{\epsilon}}\left\langle\left(\begin{array}{cc}
\alpha & 0 \\
0 & \beta
\end{array}\right) R_{\epsilon}(x) \nabla u(x), R_{\epsilon}(x) \nabla u(x)\right\rangle d x,
\end{aligned}
$$

where $E_{1}^{\epsilon} \subset E_{2}^{\epsilon}$. The set $E_{1}^{\epsilon}$ is the part of the polycrystal with isotropic conductivity $\alpha, \Omega \backslash E_{2}^{\epsilon}$ is the part of the polycrystal with isotropic conductivity $\beta$, and $E_{2}^{\epsilon} \backslash E_{1}^{\epsilon}$ is where each single crystal has an anistropic conductivity of type $\left(\begin{array}{cc}\alpha & 0 \\ 0 & \beta\end{array}\right)$, up to a rotation.

In order to describe the limit of $F_{\epsilon}$, up to the extraction of a subsequence, we may suppose that $\chi_{E_{1}^{\epsilon}} \rightarrow \theta_{1}$ and $\chi_{E_{2}^{\epsilon}} \rightarrow \theta_{2}$ weakly* in $L^{\infty}(\Omega)$, and we define $\theta=\frac{\theta_{1}+\theta_{2}}{2}$. In the notation above the value $\theta(x)$ represents the fixed limit local proportion of layers of type $\mathcal{A}$ at the point $x$, and is the analog of the local proportion of $\alpha$-connections in the discrete setting. With the classical notation of $G$-closure, we would denote by $\theta_{\alpha}$ the volume fraction of isotropic conductivity $\alpha$, by $\theta_{\beta}$ the volume fraction of isotropic conductivity $\beta$ and by $\theta_{\alpha, \beta}=1-\theta_{\alpha}-\theta_{\beta}$ the volume fraction of anisotropic conductivity. The problem we are interested in is not the classical $G$-closure of a three phase mixture, which is a very tough issue. Actually our discrete setting imposes an 
additional relationship between $\theta_{\alpha}, \theta_{\alpha}$ and $\theta_{\alpha, \beta}$, namely $\theta_{\alpha}+\frac{\theta_{\alpha, \beta}}{2}=\theta$, or equivalently $\theta_{\beta}+\frac{\theta_{\alpha, \beta}}{2}=1-\theta$, where $\theta$ is fixed by the local proportion of $\alpha$-connections. Our aim is then to characterize the $\Gamma$-limits for all values of $\theta \in[0,1]$. Surprisingly, this additional relationship trivializes the associated $G$-closure problem, which can be interpreted as a 'constrained' three-phase mixture $G$-closure problem.

The paper is organized as follows. In the second section, we give a rigorous formulation of the result that we prove in two steps: first we derive bounds on the eigenvalues of $A^{0}(x)$, then we show these bounds to be optimal. We perform a derivation of these bounds following a purely variational approach to highlight how our parameter $\theta$ intervenes in their computations, so that the optimality of those bounds is simply obtained by making the previous reasoning sharp. This proof is direct and self-contained, and can be compared with the one in the discrete setting in [6]. In the third section, we generalize the result to the uniaxial three-dimensional case. The bounds we get in the continuous case are indeed the 'trivial bounds' usually obtained in the continuum theory of bounds (for a general exposition of results connected to the computation of bounds for the effective properties of composites we refer to the monograph by Milton [11]), which may be surprising at the first glance since we deal with a three-phase mixture. The last section is dedicated to the reformulation and interpretation of the present results in terms of a constrained $G$-closure problem, where we recall the more classical proof of the trivial bounds and generalize the results.

\section{Main result and derivation of bounds in two dimensions.}

2.1. Optimal bounds. Having set the notation in the Introduction, we may describe the $\Gamma$-limits of functionals $F_{\epsilon}$ as follows.

Theorem 1. Let $F_{\epsilon}$ be defined by (1.3), let $\chi_{E_{1}^{\epsilon}} \rightarrow \theta_{1}$ and $\chi_{E_{2}^{\epsilon}} \rightarrow \theta_{2}$ weakly* in $L^{\infty}(\Omega)$, and let $\theta=\frac{\theta_{1}+\theta_{2}}{2}$. Up to the extraction of a (further) subsequence $F_{\epsilon}$ $\Gamma$-converges to an energy of the form

$$
F^{0}(u)=\int_{\Omega}\left\langle A^{0}(x) \nabla u, \nabla u\right\rangle d x
$$

on $H^{1}(\Omega)$, where for almost all $x \in \Omega$ we have $A^{0}(x) \in \mathcal{H}_{d}^{2}(\theta(x))$, and for fixed $\theta \in[0,1]$ the set $\mathcal{H}_{d}^{2}(\theta)$ is defined as the set of symmetric $2 \times 2$ matrices whose eigenvalues $\lambda_{1}, \lambda_{2}$ satisfy the bounds

$$
\alpha \leq \lambda_{1}, \lambda_{2} \leq \beta, \quad \lambda_{1}+\lambda_{2} \leq 2(\theta \alpha+(1-\theta) \beta), \quad \frac{1}{\lambda_{1}}+\frac{1}{\lambda_{2}} \leq 2\left(\frac{\theta}{\alpha}+\frac{1-\theta}{\beta}\right)
$$

Conversely, for all measurable $\theta$ and $A^{0}$ such that $A^{0}(x) \in \mathcal{H}_{d}^{2}(\theta(x))$, there exist $E_{i}^{\epsilon}$ as above such that $F_{\epsilon} \Gamma$-converges to $F^{0}$ in (2.1).

Theorem 1 states that the effective overall conductivity of the polycrystal can be characterized in terms of the local proportion of layers of type $\mathcal{A}$. As motivated in the Introduction, these bounds correspond to the bounds obtained for a square conducting lattice in [6] for diagonal matrices, or for a discrete polycrystal. With this observation in mind we have added the subscript ' $\mathrm{d}$ ' for discrete in the notation above. In terms of those bounds it must be noted that the optimal micro-geometries correspond to lattices locally oriented in the directions of the eigenvectors of the matrix $A^{0}(x)$. 
For fixed $\theta \in[0,1]$ we will denote by $\mathcal{H}_{\text {hom }}^{2}(\theta)$ the set of all homogenized matrices obtained by the homogenization of a periodic polycrystal as above with underlying volume fraction $\theta$ of $\mathcal{A}$-layers; i.e., of all matrices $B$ that satisfy the equality

$$
\begin{aligned}
& \langle B \xi, \xi\rangle=\inf \left\{\alpha \int_{E_{1}}|\xi+\nabla u|^{2} d y+\beta \int_{(0,1)^{2} \backslash E_{2}}|\xi+\nabla u|^{2} d y\right. \\
& \left.+\int_{E_{2} \backslash E_{1}}\left\langle\left(\begin{array}{cc}
\alpha & 0 \\
0 & \beta
\end{array}\right) R(y)(\xi+\nabla u(y)), R(y)(\xi+\nabla u(y))\right\rangle d y: u \in H_{\#}^{1}\left((0,1)^{2}\right)\right\},
\end{aligned}
$$

for some rotation $R(y)$, and subsets $E_{1} \subset E_{2} \subset(0,1)^{2}$, with $\left|E_{1}\right|+\left|E_{2}\right|=2 \theta$. Here and after $H_{\#}^{1}\left((0,1)^{2}\right)$ denotes the space of $H_{\text {loc }}^{1}\left(\mathbb{R}^{2}\right)$ functions that are 1-periodic in the coordinate directions. Note that the energy $\int_{\Omega}\langle B \nabla u, \nabla u\rangle d x$ corresponding to such $B$ is a particular $\Gamma$-limit of a family of the type (1.3), with $E_{i}^{\epsilon}=\epsilon E_{i}\left(E_{i}\right.$ are extended by 1-periodicity) and $R_{\epsilon}(y)=R(y / \epsilon)$. The triplet $\left(E_{1}, E_{2}, R\right)$ is called the (micro-)geometry of the corresponding periodic polycrystal.

First, note that by a general compactness result for the $\Gamma$-convergence of quadratic forms ([5], [3], [7], [4] Theorem 4.13) we obtain that, up to subsequences, $F_{\epsilon} \Gamma$ converges to some $F^{0}$ represented as in (2.1) for some $A^{0}(x)$. By the well known localization principle (see [4] Section 5.4.1 for a simple version, or [14] for a slightly more general one), we have that for almost every $x \in \Omega$ and for all $\xi \in \mathbb{R}^{2}$,

$$
A^{0}(x) \in \mathcal{H}_{\text {hom }}^{2}(\theta(x)+o(1))+o(1) ;
$$

i.e., for every $\eta>0$ there exist $R, E_{1}$, and $E_{2}$, with ||$E_{1}|+| E_{2}|-2 \theta(x)|<\eta$, such that if $B$ is as above then $\left|B-A^{0}(x)\right| \leq o(1)$ as $\eta \rightarrow 0$.

In the rest of the section we will prove that $\mathcal{H}_{\text {hom }}^{2}(\theta)=\mathcal{H}_{d}^{2}(\theta)$. From this inequality, the continuity of the bounds in (2.2), and the closure of $\mathcal{H}_{d}^{2}(\theta)$, it then follows that $A^{0}(x) \in \mathcal{H}_{d}^{2}(\theta(x))=\mathcal{H}_{\text {hom }}^{2}(\theta(x))$ for a.a. $x \in \Omega$ and the first part of Theorem 1. We will therefore fix $E_{1}, E_{2}$, and $R$ as in (2.3), and introduce a rotation angle $\phi$. so that we can write $R$ as

$$
R(y)=\left(\begin{array}{cc}
\cos (\phi(y)) & \sin (\phi(y)) \\
-\sin (\phi(y)) & \cos (\phi(y))
\end{array}\right) .
$$

Note that the set $\mathcal{H}_{\mathrm{hom}}^{2}(\theta)$ is invariant by multiplication by rotations. Since it is also composed of symmetric matrices, this set is consequently characterized by the set of all possible eigenvalues of such matrices. The bounds are therefore derived in the plane of the eigenvalues $\left(\lambda_{1}, \lambda_{2}\right)$ of matrices in $\mathcal{H}_{\mathrm{hom}}^{2}(\theta)$.

\subsection{Simple bounds.}

Proposition 2.1. For $i=1,2$ we have $\alpha \leq \lambda_{i} \leq \beta$.

These easy bounds follow immediately by the pointwise estimate $\alpha \operatorname{Id} \leq A_{\epsilon}(y) \leq$ $\beta$ Id.

2.3. Bounds from arithmetic means.

Proposition 2.2. We have

$$
\lambda_{1}+\lambda_{2} \leq 2(\alpha \theta+(1-\theta) \beta) .
$$


Proof. Take $\xi=e_{i}$ and the test function $u=0$ in (2.3). We then have for $i=1,2$

$$
\lambda_{i} \leq \alpha\left|E_{1}\right|+\beta\left(1-\left|E_{2}\right|\right)+\int_{E_{2} \backslash E_{1}}\left\langle\left(\begin{array}{cc}
\alpha & 0 \\
0 & \beta
\end{array}\right) R(y) e_{i}, R(y) e_{i}\right\rangle d y
$$

In addition, since $\left\{e_{1}, e_{2}\right\}$ is an orthonormal basis of $\mathbb{R}^{2}$ and $R(y)$ is a rotation, for almost every $y \in E_{2} \backslash E_{1}$ we have

$$
\left\langle\left(\begin{array}{cc}
\alpha & 0 \\
0 & \beta
\end{array}\right) R(y) e_{1}, R(y) e_{1}\right\rangle+\left\langle\left(\begin{array}{cc}
\alpha & 0 \\
0 & \beta
\end{array}\right) R(y) e_{2}, R(y) e_{2}\right\rangle=\alpha+\beta .
$$

Indeed the first term of (2.7) reads $\alpha \cos ^{2} \phi+\beta \sin ^{2} \phi$ and the second term $\beta \cos ^{2} \phi+$ $\alpha \sin ^{2} \phi$. The combination of (2.6) and (2.7) gives

$$
\lambda_{1}+\lambda_{2} \leq 2 \alpha\left|E_{1}\right|+2 \beta\left(1-\left|E_{2}\right|\right)+\left(\left|E_{2}\right|-\left|E_{1}\right|\right)(\alpha+\beta),
$$

which yields (2.5) using $\theta=\frac{\left|E_{2}\right|+\left|E_{1}\right|}{2}$.

\subsection{Bounds from harmonic means.}

Proposition 2.3. We have

$$
\frac{1}{\lambda_{1}}+\frac{1}{\lambda_{2}} \leq 2\left(\frac{\theta}{\alpha}+\frac{1-\theta}{\beta}\right) .
$$

The rest of the section is devoted to proving this bound, relying on two arguments. The first one is a slicing argument, which is based on the simple inequality $|\nabla u|^{2} \geq$ $\left(\partial_{i} u\right)^{2}$ and allows to reduce to one-dimensional problems. The second tool is some convexity inequality.

Slicing argument. We first prove the following inequalities:

$$
\begin{aligned}
\lambda_{1} \geq & \int_{0}^{1}\left(\frac{1}{\alpha}\left|E_{1}^{1}\left(y_{2}\right)\right|+\frac{1}{\beta}\left(1-\left|E_{2}^{1}\left(y_{2}\right)\right|\right)\right. \\
& \left.+\int_{E_{2}^{1}\left(y_{2}\right) \backslash E_{1}^{1}\left(y_{2}\right)} \frac{\alpha \sin ^{2} \phi(y)+\beta \cos ^{2} \phi(y)}{\alpha \beta} d y_{1}\right)^{-1} d y_{2}, \\
\lambda_{2} \geq & \int_{0}^{1}\left(\frac{1}{\alpha}\left|E_{1}^{2}\left(y_{1}\right)\right|+\frac{1}{\beta}\left(1-\left|E_{2}^{2}\left(y_{1}\right)\right|\right)\right. \\
& \left.+\int_{E_{2}^{2}\left(y_{1}\right) \backslash E_{1}^{2}\left(y_{1}\right)} \frac{\beta \sin ^{2} \phi(y)+\alpha \cos ^{2} \phi(y)}{\alpha \beta} d y_{2}\right)^{-1} d y_{1},
\end{aligned}
$$

where

$$
\begin{array}{ll}
E_{1}^{1}\left(y_{2}\right)=\left\{y_{1} \in(0,1), \chi_{E_{1}}\left(y_{1}, y_{2}\right)=1\right\}, \quad E_{2}^{1}\left(y_{2}\right)=\left\{y_{1} \in(0,1), \chi_{E_{2}}\left(y_{1}, y_{2}\right)=1\right\} \\
E_{1}^{2}\left(y_{1}\right)=\left\{y_{2} \in(0,1), \chi_{E_{1}}\left(y_{1}, y_{2}\right)=1\right\}, \quad E_{2}^{2}\left(y_{1}\right)=\left\{y_{2} \in(0,1), \chi_{E_{2}}\left(y_{1}, y_{2}\right)=1\right\}
\end{array}
$$

and $\chi_{E}$ denotes the characteristic function of the set $E$. These sets satisfy

$$
\int_{0}^{1}\left|E_{1}^{1}\left(y_{2}\right)\right| d y_{2}=\int_{0}^{1}\left|E_{1}^{2}\left(y_{1}\right)\right| d y_{1}=\theta_{1} \text { and } \int_{0}^{1}\left|E_{2}^{1}\left(y_{2}\right)\right| d y_{2}=\int_{0}^{1}\left|E_{2}^{2}\left(y_{1}\right)\right| d y_{1}=\theta_{2} .
$$

To get (2.10), we first prove the following result. 
Proposition 2.4. For all $\left(u_{1}, u_{2}\right) \in \mathbb{R}^{2}$ and $\phi \in(0,2 \pi)$, we have

$$
\begin{aligned}
& \left\langle\left(\begin{array}{cc}
\alpha & 0 \\
0 & \beta
\end{array}\right) R(\phi)\left(u_{1} e_{1}+u_{2} e_{2}\right), R(\phi)\left(u_{1} e_{1}+u_{2} e_{2}\right)\right\rangle \geq u_{1}^{2} \frac{\alpha \beta}{\alpha \sin ^{2} \phi+\beta \cos ^{2} \phi}, \\
& \left\langle\left(\begin{array}{cc}
\alpha & 0 \\
0 & \beta
\end{array}\right) R(\phi)\left(u_{1} e_{1}+u_{2} e_{2}\right), R(\phi)\left(u_{1} e_{1}+u_{2} e_{2}\right)\right\rangle \geq u_{2}^{2} \frac{\alpha \beta}{\beta \sin ^{2} \phi+\alpha \cos ^{2} \phi},
\end{aligned}
$$

where $\left\{e_{1}, e_{2}\right\}$ is the canonical basis of $\mathbb{R}^{2}$.

Proof. It is enough to prove (2.11). Denote by $\chi_{\phi}\left(u_{1}, u_{2}\right)$ the left-hand side of $(2.11)$. A direct calculation shows that

$$
\chi_{\phi}\left(u_{1}, u_{2}\right)=\alpha\left(u_{1} \cos \phi-u_{2} \sin \phi\right)^{2}+\beta\left(u_{1} \sin \phi+u_{2} \cos \phi\right)^{2} .
$$

At fixed $u_{1}, v \mapsto \chi_{\phi}\left(u_{1}, v\right)$ is quadratic and convex. This function attains its minimum on $\mathbb{R}$, at

$$
v_{2}=u_{1} \frac{\alpha-\beta}{\alpha \sin ^{2} \phi+\beta \cos ^{2} \phi} \sin \phi \cos \phi .
$$

We then have

$$
\begin{aligned}
\chi_{\phi}\left(u_{1}, v_{2}\right) & =u_{1}^{2}\left(\alpha \cos ^{2} \phi+\beta \sin ^{2} \phi-\frac{(\beta-\alpha)^{2} \sin ^{2} \phi \cos ^{2} \phi}{\alpha \sin ^{2} \phi+\beta \cos ^{2} \phi}\right) \\
& =u_{1}^{2} \frac{\alpha \beta\left(\cos ^{2} \phi+\sin ^{2} \phi\right)^{2}}{\alpha \sin ^{2} \phi+\beta \cos ^{2} \phi} \\
& =u_{1}^{2} \frac{\alpha \beta}{\alpha \sin ^{2} \phi+\beta \cos ^{2} \phi}
\end{aligned}
$$

as desired.

We now integrate in the $e_{2}$ direction. If $u$ is such that $\nabla u=e_{1}+\nabla w, w \in$ $H_{\#}^{1}((0,1))$, we then have

$$
\begin{aligned}
\lambda_{1}= & \int_{0}^{1}\left(\alpha \int_{E_{1}^{1}\left(y_{2}\right)}|\nabla u|^{2} d y_{1}+\beta \int_{(0,1) \backslash E_{2}^{1}\left(y_{2}\right)}|\nabla u|^{2} d y_{1}\right. \\
& \left.+\int_{E_{2}^{1}\left(y_{2}\right) \backslash E_{1}^{1}\left(y_{2}\right)}\left\langle\left(\begin{array}{cc}
\alpha & 0 \\
0 & \beta
\end{array}\right) R(y) \nabla u, R(y) \nabla u\right\rangle d y_{1}\right) d y_{2} \\
\geq & \int_{0}^{1}\left(\alpha \int_{E_{1}^{1}\left(y_{2}\right)} \partial_{1} u^{2} d y_{1}+\beta \int_{(0,1) \backslash E_{2}^{1}\left(y_{2}\right)} \partial_{1} u^{2} d y_{1}\right. \\
& \left.+\int_{E_{2}^{1}\left(y_{2}\right) \backslash E_{1}^{1}\left(y_{2}\right)} d y_{1} \frac{\partial_{1} u^{2} \alpha \beta}{\alpha \sin ^{2} \phi(y)+\beta \cos ^{2} \phi(y)}\right) d y_{2} \\
\geq & \int_{0}^{1} \inf \left\{\alpha \int_{E_{1}^{1}\left(y_{2}\right)} v^{\prime}\left(y_{1}\right)^{2} d y_{1}+\beta \int_{(0,1) \backslash E_{2}^{1}\left(y_{2}\right)} v^{\prime}\left(y_{1}\right)^{2} d y_{1}\right. \\
& \left.+\int_{E_{2}^{1}\left(y_{2}\right) \backslash E_{1}^{1}\left(y_{2}\right)} \frac{v^{\prime}\left(y_{1}\right)^{2} \alpha \beta}{\alpha \sin ^{2} \phi(y)+\beta \cos ^{2} \phi(y)} d y_{1}: v \in H_{0}^{1}((0,1))\right\} d y_{2} .
\end{aligned}
$$

The infimum in the integral of (2.13) can be explicitly computed by using the following well-known result. 
Proposition 2.5. Let $f \in L^{\infty}((0,1))$ be such that inf $f>0$, then

$\inf \left\{\int_{0}^{1} f(y) v^{\prime}(y)^{2}: v \in H^{1}(0,1), v(0)=0, v(1)=1\right\}=\left(\int_{0}^{1} \frac{d y}{f(y)}\right)^{-1}$

Inserting formula (2.14) in (2.13), we obtain

$$
\begin{aligned}
\lambda_{1} \geq \int_{0}^{1} & \left(\frac{1}{\alpha}\left|E_{1}^{1}\left(y_{2}\right)\right|+\frac{1}{\beta}\left(1-\left|E_{2}^{1}\left(y_{2}\right)\right|\right)\right. \\
& \left.+\int_{E_{2}^{1}\left(y_{2}\right) \backslash E_{1}^{1}\left(y_{2}\right)} \frac{\alpha \sin ^{2} \phi(y)+\beta \cos ^{2} \phi(y)}{\alpha \beta} d y_{1}\right)^{-1} d y_{2} .
\end{aligned}
$$

The same proof holds for $\lambda_{2}$.

To conclude the proof of the bounds, we use some convexity properties.

A convexity inequality. Proposition 2.6. Let $b>a>0, c>0, \gamma>0$ and $f \in L^{1}\left((a, b), \mathbb{R}^{+}\right)$, then

$$
\int_{a}^{b} \frac{d y}{c+f(y)} \geq \frac{(b-a)^{2}}{\int_{a}^{b}(c+f(y)) d y}
$$

This result just amounts to stating that the harmonic mean is lower than the arithmetic mean, and it is immediately obtained by Hölder's inequality.

Applying Proposition 2.6 to (2.10), for $a=0, b=1$ and $c=1 / \alpha$, we obtain

$$
\begin{aligned}
\lambda_{1} \geq & {\left[\int _ { 0 } ^ { 1 } \left(\frac{1}{\alpha}\left|E_{1}^{1}\left(y_{2}\right)\right|+\frac{1}{\beta}\left(1-\left|E_{2}^{1}\left(y_{2}\right)\right|\right)\right.\right.} \\
& \left.\left.+\int_{E_{2}^{1}\left(y_{2}\right) \backslash E_{1}^{1}\left(y_{2}\right)} \frac{\alpha \sin ^{2} \phi(y)+\beta \cos ^{2} \phi(y)}{\alpha \beta} d y_{1}\right) d y_{2}\right]^{-1} \\
= & \left(\frac{\theta_{1}}{\alpha}+\frac{1-\theta_{2}}{\beta}+\int_{E_{2} \backslash E_{1}} \frac{\alpha \sin ^{2} \phi(y)+\beta \cos ^{2} \phi(y)}{\alpha \beta} d y\right)^{-1} \\
\lambda_{2} \geq & {\left[\int _ { 0 } ^ { 1 } \left(\frac{1}{\alpha}\left|E_{1}^{2}\left(y_{1}\right)\right|+\frac{1}{\beta}\left(1-\left|E_{2}^{2}\left(y_{1}\right)\right|\right)\right.\right.} \\
& \left.\left.+\int_{E_{2}^{2}\left(y_{1}\right) \backslash E_{1}^{2}\left(y_{1}\right)} \frac{\beta \sin ^{2} \phi(y)+\alpha \cos ^{2} \phi(y)}{\alpha \beta} d y_{2}\right) d y_{1}\right]^{-1} \\
= & \left(\frac{\theta_{1}}{\alpha}+\frac{1-\theta_{2}}{\beta}+\int_{E_{2} \backslash E_{1}} \frac{\beta \sin ^{2} \phi(y)+\alpha \cos ^{2} \phi(y)}{\alpha \beta} d y\right)^{-1}
\end{aligned}
$$

Therefore we have

$$
\begin{aligned}
\frac{1}{\lambda_{1}}+\frac{1}{\lambda_{2}} & \leq 2 \frac{\theta_{1}}{\alpha}+2 \frac{1-\theta_{2}}{\beta}+\int_{E_{2} \backslash E_{1}}\left(\frac{\alpha \cos ^{2} \phi+\beta \sin ^{2} \phi}{\alpha \beta}\right. \\
& \left.=2 \frac{\beta \cos ^{2} \phi+\alpha \sin ^{2} \phi}{\alpha \beta}\right) d y \\
& =2 \frac{\theta_{1}}{\beta}+\left(\theta_{2}-\theta_{1}\right)\left(\frac{1}{\alpha}+\frac{1}{\beta}\right)
\end{aligned}
$$

which exactly reads as (2.9). 
3. Optimality of the bounds. We now prove the last statement of Theorem 1 . By approximation with piecewise-constant functions, it is sufficient to consider the case of $\theta$ and $A^{0}$ constant; i.e., it will be sufficient to show that for all symmetric $A^{0}$ with eigenvalues satisfying (2.2) we find a suitable geometry such that each $\left\langle A^{0} \xi, \xi\right\rangle$ is represented as a minimum problem as in (2.3). By rotational invariance, it suffices to consider diagonal $A^{0}$. Furthermore, it is not restrictive to treat the case of eigenvalues belonging to the boundary of the set defined by (2.3) only (the 'extremal' cases). In fact, using a well-known "lamination formula" (see [17, 16] Proposition 3) we may easily construct geometries for a generic diagonal $A^{0}$ using those 'extremal geometries'.

We will consider polycrystals $(0,1)^{2}$ defined by $A(y)=\left(\begin{array}{cc}a_{1}(y) & 0 \\ 0 & a_{2}(y)\end{array}\right)$ for $y \in(0,1)^{2}$, and the corresponding homogenized $A^{0}$, defined by

$$
\left\langle A^{0} \xi, \xi\right\rangle=\inf \left\{\int_{(0,1)^{2}}\langle A(y)(\xi+\nabla u(y)), \xi+\nabla u(y)\rangle, u \in H_{\#}^{1}\left((0,1)^{2}\right)\right\}
$$

for all $\xi \in \mathbb{R}^{2}$. Note that in terms of the micro-geometry of the polycrystal the rotation $R$ is either the identity or a rotation of ninety degrees.

3.1. Arithmetic bound. Let $\theta_{1} \in(0,1)$ and $\theta_{2} \in(0,1)$. We define $A(y)$ by $a_{1}(y)=\alpha$ if $y_{2} \leq \theta_{1}, a_{1}(y)=\beta$ if $y_{2}>\theta_{1}$, and $a_{2}(y)=\alpha$ if $y_{1} \leq \theta_{2}, a_{2}(y)=\beta$ if $y_{1}>\theta_{2}$.

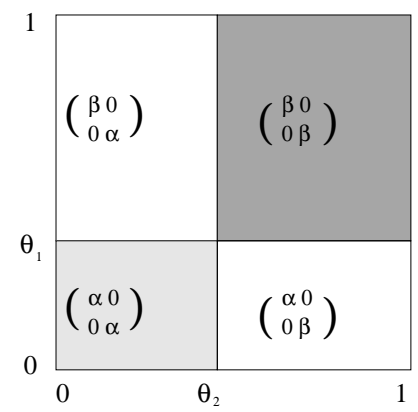

FIG. 3.1. Optimal geometry for the arithmetic bound

We will then prove that $A^{0}=\left(\begin{array}{cc}\bar{a}\left(\theta_{1}\right) & 0 \\ 0 & \bar{a}\left(\theta_{2}\right)\end{array}\right)$, where $\bar{a}(\lambda)=\alpha \lambda+\beta(1-\lambda)$.

Testing with $u=0$ in (3.1), we obtain $\left\langle A^{0} \xi, \xi\right\rangle \leq \bar{a}\left(\theta_{1}\right) \xi_{1}^{2}+\bar{a}\left(\theta_{2}\right) \xi_{2}^{2}$. To prove the converse inequality we use the slicing method, obtaining

$$
\begin{aligned}
& \int_{(0,1)^{2}}\langle A(y)(\xi+\nabla u(y)), \xi+\nabla u(y)\rangle=\int_{0}^{1}\left(\int_{0}^{\theta_{1}} \alpha\left(\xi_{1}+\partial_{1} u\left(y_{1}, y_{2}\right)\right)^{2} d y_{2}\right) d y_{1} \\
& +\int_{0}^{1}\left(\int_{\theta_{1}}^{1} \beta\left(\xi_{1}+\partial_{1} u\left(y_{1}, y_{2}\right)\right)^{2} d y_{2}\right) d y_{1}+\int_{0}^{1}\left(\int_{0}^{\theta_{2}} \alpha\left(\xi_{2}+\partial_{2} u\left(y_{1}, y_{2}\right)\right)^{2} d y_{1}\right) d y_{2} \\
& +\int_{0}^{1}\left(\int_{\theta_{2}}^{1} \beta\left(\xi_{2}+\partial_{2} u\left(y_{1}, y_{2}\right)\right)^{2} d y_{1}\right) d y_{2} .
\end{aligned}
$$

Switching the order of the integrals and minimizing each term of the right-hand side 
of (3.2) separately yield the desired inequality, and thus the equality

$$
\left\langle A^{0} \xi, \xi\right\rangle=\bar{a}\left(\theta_{1}\right) \xi_{1}^{2}+\bar{a}\left(\theta_{2}\right) \xi_{2}^{2} .
$$

If $\theta_{2}+\theta_{1}=2 \theta$ then $\bar{a}\left(\theta_{1}\right)+\bar{a}\left(\theta_{2}\right)=\alpha \theta+\beta(1-\theta)$, which is the arithmetic bound.

3.2. Harmonic bound. For the harmonic bound, let us consider $a_{1}(y)=\alpha$ if $y_{1} \leq \theta_{1}, a_{1}(y)=\beta$ if $y_{1}>\theta_{1}$, and $a_{2}(y)=\alpha$ if $y_{2} \leq \theta_{2}, a_{2}(y)=\beta$ if $y_{2}>\theta_{2}$.

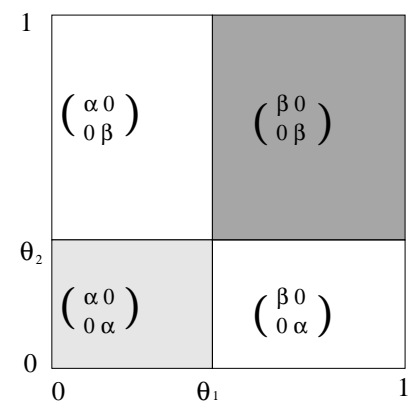

FIG. 3.2. Optimal geometry for the harmonic bound

We will then prove that $A^{0}=\left(\begin{array}{cc}\underline{a}\left(\theta_{1}\right) & 0 \\ 0 & \underline{a}\left(\theta_{2}\right)\end{array}\right)$, where $\underline{a}(\lambda)=\left(\frac{\lambda}{\alpha}+\frac{1-\lambda}{\beta}\right)^{-1}$.

Let $u_{1}:(0,1) \mapsto \mathbb{R}$ be the one-dimensional minimizer of

$\inf \left\{\int_{0}^{\theta_{1}} \alpha\left(\xi_{1}+v^{\prime}(z)\right)^{2} d z+\int_{\theta_{1}}^{1} \beta\left(\xi_{1}+v^{\prime}(z)\right)^{2} d z: v(0)=v(1)=0\right\}=\underline{a}\left(\theta_{1}\right) \xi_{1}^{2}$,

and $u_{2}:(0,1) \mapsto \mathbb{R}$ be the one-dimensional minimizer of

$$
\inf \left\{\int_{0}^{\theta_{2}} \alpha\left(\xi_{2}+v^{\prime}(z)\right)^{2} d z+\int_{\theta_{2}}^{1} \beta\left(\xi_{2}+v^{\prime}(z)\right)^{2} d z: v(0)=v(1)=0\right\}=\underline{a}\left(\theta_{2}\right) \xi_{2}^{2} .
$$

Let $u(y)=u_{1}\left(y_{1}\right)+u_{2}\left(y_{2}\right)$ be a test function in (3.1). We then have

$$
\left\langle A^{0} \xi, \xi\right\rangle \leq \underline{a}\left(\theta_{1}\right) \xi_{1}^{2}+\underline{a}\left(\theta_{2}\right) \xi_{2}^{2} .
$$

Once more we prove the converse inequality using the slicing argument.

$$
\begin{aligned}
\int_{(0,1)^{2}}\langle A(y) & (\xi+\nabla u(y)), \xi+\nabla u(y)\rangle= \\
& \int_{0}^{1}\left(\int_{0}^{\theta_{1}} \alpha\left(\xi_{1}+\partial_{1} u\left(y_{1}, y_{2}\right)\right)^{2} d y_{1}+\int_{\theta_{1}}^{1} \beta\left(\xi_{1}+\partial_{1} u\left(y_{1}, y_{2}\right)\right)^{2} d y_{1}\right) d y_{2} \\
+ & \int_{0}^{1}\left(\int_{0}^{\theta_{2}} \alpha\left(\xi_{2}+\partial_{2} u\left(y_{1}, y_{2}\right)\right)^{2} d y_{2}+\int_{\theta_{2}}^{1} \beta\left(\xi_{2}+\partial_{2} u\left(y_{1}, y_{2}\right)\right)^{2} d y_{2}\right) d y_{1}
\end{aligned}
$$

We obtain the desired inequality by optimizing pointwise the integrands of the right handside of (3.3).

If $\theta_{2}+\theta_{1}=2 \theta$ then $\frac{1}{\underline{a}\left(\theta_{1}\right)}+\frac{1}{\underline{a}\left(\theta_{2}\right)}=\frac{\theta}{\alpha}+\frac{1-\theta}{\beta}$, which is the harmonic bound. 
3.3. Optimal bounds. The simple bounds $\lambda_{i}=\alpha, \beta$ can always be reached by layering. Depending on $\theta$, the shape of the optimal bounds may vary (see Figure 5 in [6], and the details therein). Once the arithmetic and harmonic bounds are shown to be attained, the convex set delimited by these bounds and the simple bounds can also be attained by layering.

4. Extension to higher dimension. Theorem 1 has a natural counterpart in higher dimension. We only consider the physically meaningful three-dimensional case, and especially the uniaxial basic crystal. In this case we deal with the integral functionals

$$
\begin{aligned}
F_{\epsilon}(u)=\alpha \int_{E_{1}^{\epsilon}} & |\nabla u|^{2}+\beta \int_{\Omega \backslash E_{2}^{\epsilon}}|\nabla u|^{2} \\
& +\int_{E_{3}^{\epsilon}}\left\langle\left(\begin{array}{ccc}
\alpha & 0 & 0 \\
0 & \beta & 0 \\
0 & 0 & \beta
\end{array}\right) R_{\epsilon}(x) \nabla u(x), R_{\epsilon}(x) \nabla u(x)\right\rangle d x \\
& +\int_{\left(E_{2}^{\epsilon} \backslash E_{1}^{\epsilon}\right) \backslash E_{3}^{\epsilon}}\left\langle\left(\begin{array}{ccc}
\beta & 0 & 0 \\
0 & \alpha & 0 \\
0 & 0 & \alpha
\end{array}\right) R_{\epsilon}(x) \nabla u(x), R_{\epsilon}(x) \nabla u(x)\right\rangle d x
\end{aligned}
$$

where $E_{1}^{\epsilon} \subset E_{2}^{\epsilon}, E_{3}^{\epsilon} \subset E_{2}^{\epsilon} \backslash E_{1}^{\epsilon}$ and $R_{\epsilon}$ is a rotation.

As in the two-dimensional case, in order to describe the $\Gamma$-limit of $F_{\epsilon}$, up to the extraction of a subsequence, we may suppose that $\chi_{E_{1}^{\epsilon}} \rightarrow \theta_{1}, \chi_{E_{2}^{\epsilon}} \rightarrow \theta_{2}$, and $\chi_{E_{3}^{\epsilon}} \rightarrow \theta_{3}$ weakly* in $L^{\infty}(\Omega)$, and we define $\theta=\frac{\theta_{1}+2 \theta_{2}-\theta_{3}}{3}$, again the value $\theta(x)$ representing the fixed limit local proportion of layers of type $\mathcal{A}$ at the point $x$.

Theorem 2. Let $F_{\epsilon}$ be defined by $(4.1)$, let $\chi_{E_{i}^{\epsilon}} \rightarrow \theta_{i}$ weakly in $L^{\infty}(\Omega)$, and let $\theta=\frac{\theta_{1}+2 \theta_{2}-\theta_{3}}{3}$. Up to the extraction of a (further) subsequence $F_{\epsilon} \Gamma$-converges to an energy of the form

$$
F^{0}(u)=\int_{\Omega}\left\langle A^{0}(x) \nabla u, \nabla u\right\rangle d x
$$

on $H^{1}(\Omega)$, where for almost all $x \in \Omega$ we have $A^{0}(x) \in \mathcal{H}_{d}^{3}(\theta(x))$, where for fixed $\theta \in[0,1]$ the set $\mathcal{H}_{d}^{3}(\theta)$ is defined as the set of symmetric $3 \times 3$ matrices whose eigenvalues $\lambda_{1}, \lambda_{2}, \lambda_{3}$ satisfy the bounds

$$
\alpha \leq \lambda_{1}, \lambda_{2}, \lambda_{3} \leq \beta, \quad \lambda_{1}+\lambda_{2}+\lambda_{3} \leq 3 \bar{a}(\theta), \quad \frac{1}{\lambda_{1}}+\frac{1}{\lambda_{2}}+\frac{1}{\lambda_{3}} \leq \frac{3}{\underline{a}(\theta)}
$$

Conversely, for all measurable $\theta$ and $A^{0}$ such that $A^{0}(x) \in \mathcal{H}_{d}^{3}(\theta(x))$, there exist $E_{i}^{\epsilon}$ as above such that $F_{\epsilon} \Gamma$-converges to $F^{0}$ in (4.2).

We now adapt Sections 2 and 3 to prove Theorem 2.

Since the anisotropic conductivity matrices only have two eigenvalues $\alpha$ and $\beta$, in order to diagonalize the matrix it suffices to determine the eigenvector associated to the eigenvalue of multiplicity 1 , the orthogonal subspace being an eigensubspace associated to the other eigenvalue. Therefore, we only need two angles for the rotations which can be written as

$$
R(\phi, \psi)=\left(\begin{array}{ccc}
\sin \psi & \cos \phi \cos \psi & \sin \phi \cos \psi \\
\cos \psi & -\cos \phi \sin \psi & -\sin \phi \sin \psi \\
0 & -\sin \phi & \cos \phi
\end{array}\right)
$$


The calculations are more involved in the three-dimensional case. We leave the details to the interested reader and only give the intermediate results.

4.1. Bounds from arithmetic means. Direct calculations give

$$
\begin{aligned}
& \left\langle\left(\begin{array}{ccc}
\alpha & 0 & 0 \\
0 & \beta & 0 \\
0 & 0 & \beta
\end{array}\right) R(\phi, \psi) e_{1}, R(\phi, \psi) e_{1}\right\rangle=\alpha \sin ^{2} \psi+\beta \cos ^{2} \psi \\
& \left\langle\left(\begin{array}{lll}
\alpha & 0 & 0 \\
0 & \beta & 0 \\
0 & 0 & \beta
\end{array}\right) R(\phi, \psi) e_{2}, R(\phi, \psi) e_{2}\right\rangle=\alpha \cos ^{2} \phi \cos ^{2} \psi+\beta\left(\cos ^{2} \phi \sin ^{2} \psi+\sin ^{2} \phi\right) \\
& \left\langle\left(\begin{array}{lll}
\alpha & 0 & 0 \\
0 & \beta & 0 \\
0 & 0 & \beta
\end{array}\right) R(\phi, \psi) e_{3}, R(\phi, \psi) e_{3}\right\rangle=\alpha \sin ^{2} \phi \cos ^{2} \psi+\beta\left(\sin ^{2} \phi \sin ^{2} \psi+\cos ^{2} \phi\right),
\end{aligned}
$$

whose sum is $\alpha+2 \beta$. The calculation for the other anisotropic conductivity matrix yields $\beta+2 \alpha$. Therefore,

$$
\begin{aligned}
\lambda_{1}+\lambda_{2}+\lambda_{3} & \leq 3 \theta_{1} \alpha+3\left(1-\theta_{2}\right) \beta+\theta_{3}(\alpha+2 \beta)+\left(\theta_{2}-\theta_{1}-\theta_{3}\right)(2 \alpha+\beta) \\
& =3(\alpha \theta+(1-\theta) \beta)
\end{aligned}
$$

which is the desired arithmetic bound.

4.2. Bounds from harmonic means. For convenience, we will make use of c for cos, $\mathrm{s}$ for $\sin$, and $v=\left(v_{1}, v_{2}, v_{3}\right)$ in the sequel. We set

$$
\begin{aligned}
H\left(v_{1}, v_{2}, v_{3}\right):=\left\langle\left(\begin{array}{ccc}
\alpha & 0 & 0 \\
0 & \beta & 0 \\
0 & 0 & \beta
\end{array}\right) R(\phi, \psi) v, R(\phi, \psi) v\right\rangle \\
=\alpha\left(v_{1} \mathrm{~s}_{\psi}+v_{2} \mathrm{c}_{\phi} \mathrm{c}_{\psi}+v_{3} \mathrm{~s}_{\phi} \mathrm{c}_{\phi}\right)^{2}+\beta\left(v_{1} \mathrm{c}_{\psi}-v_{2} \mathrm{c}_{\phi} \mathrm{s}_{\psi}-v_{3} \mathrm{~s}_{\phi} \mathrm{s}_{\psi}\right)^{2} \\
+\beta\left(-v_{2} \mathrm{~s}_{\phi}+v_{3} \mathrm{c}_{\phi}\right)^{2}
\end{aligned}
$$

As in the two-dimensional case, we optimize $H$ on $\mathbb{R}^{2}$, one variable being fixed. These minimizers exist since $H$ is a symmetric positive definite quadratic form. The first derivatives of $H$ with respect to $v_{1}, v_{2}, v_{3}$ read as

$$
\begin{gathered}
\partial_{1} H\left(v_{1}, v_{2}, v_{3}\right)=v_{1}\left(\alpha \mathrm{s}_{\psi}^{2}+\beta \mathrm{c}_{\psi}^{2}\right)+v_{2} \mathrm{c}_{\phi} \mathrm{c}_{\psi} \mathrm{s}_{\psi}(\alpha-\beta)+v_{3} \mathrm{~s}_{\phi} \mathrm{c}_{\psi} \mathrm{s}_{\psi}(\alpha-\beta) \\
\partial_{2} H\left(v_{1}, v_{2}, v_{3}\right)=v_{1}(\alpha-\beta) \mathrm{c}_{\phi} \mathrm{c}_{\psi} \mathrm{s}_{\psi}+v_{2}\left(\alpha \mathrm{c}_{\phi}^{2} \mathrm{c}_{\psi}^{2}+\beta \mathrm{c}_{\phi}^{2} \mathrm{c}_{\psi}^{2}+\beta \mathrm{s}_{\phi}^{2}\right) \\
\quad+v_{3}(\alpha-\beta) \mathrm{c}_{\psi}^{2} \mathrm{c}_{\phi} \mathrm{s}_{\phi} \\
\partial_{3} H\left(v_{1}, v_{2}, v_{3}\right)=v_{1}(\alpha-\beta) \mathrm{s}_{\phi} \mathrm{c}_{\psi} \mathrm{s}_{\psi}+v_{2}(\alpha-\beta) \mathrm{c}_{\psi}^{2} \mathrm{c}_{\phi} \mathrm{s}_{\phi}+v_{3}\left(\alpha \mathrm{s}_{\phi}^{2} \mathrm{c}_{\psi}^{2}\right. \\
\left.+\beta \mathrm{s}_{\phi}^{2} \mathrm{~s}_{\psi}^{2}+\beta \mathrm{c}_{\phi}^{2}\right)
\end{gathered}
$$

We treat three cases, at fixed $u_{1}, u_{2}$ and $u_{3}$, respectively.

Case 1. We minimize $H\left(u_{1}, \cdot, \cdot\right)$ on $\mathbb{R}^{2}$. This is equivalent to solving

$$
\left\{\begin{array}{l}
\partial_{2} H\left(u_{1}, v_{2}, v_{3}\right)=0 \\
\partial_{3} H\left(u_{1}, v_{2}, v_{3}\right)=0
\end{array}\right.
$$


The solution is given by

$$
\begin{aligned}
& v_{2}=u_{1}(\beta-\alpha) \frac{\mathrm{c}_{\phi} \mathrm{c}_{\psi} \mathrm{s}_{\psi}}{\alpha \mathrm{c}_{\psi}^{2}+\beta \mathrm{s}_{\psi}^{2}} \\
& v_{3}=u_{1}(\beta-\alpha) \frac{\mathrm{s}_{\phi} \mathrm{c}_{\psi} \mathrm{s}_{\psi}}{\alpha \mathrm{c}_{\psi}^{2}+\beta \mathrm{s}_{\psi}^{2}} .
\end{aligned}
$$

The minimum then reads

$$
\begin{aligned}
\inf _{w_{2}, w_{3}} H\left(u_{1}, w_{2}, w_{3}\right) & =H\left(u_{1}, v_{2}, v_{3}\right) \\
& =\alpha \beta \frac{u_{1}^{2}}{\left(\alpha \mathrm{c}_{\psi}^{2}+\beta \mathrm{s}_{\psi}^{2}\right)^{2}}\left(\beta \mathrm{s}_{\psi}^{2}+\alpha \mathrm{c}_{\psi}^{2}\right) \\
& =u_{1}^{2} \frac{\alpha \beta}{\alpha \mathrm{c}_{\psi}^{2}+\beta \mathrm{s}_{\psi}^{2}} .
\end{aligned}
$$

Case 2. We minimize $H\left(\cdot, u_{2}, \cdot\right)$ on $\mathbb{R}^{2}$. This is equivalent to solving

$$
\left\{\begin{array}{l}
\partial_{1} H\left(v_{1}, u_{2}, v_{3}\right)=0 \\
\partial_{3} H\left(v_{1}, u_{2}, v_{3}\right)=0
\end{array}\right.
$$

The solution is given by

$$
\begin{aligned}
& v_{1}=u_{2}(\beta-\alpha) \frac{\mathrm{c}_{\psi} \mathrm{c}_{\phi} \mathrm{s}_{\psi}}{\mathrm{c}_{\phi}^{2}\left(\alpha \mathrm{s}_{\psi}^{2}+\beta \mathrm{c}_{\psi}^{2}\right)+\alpha \mathrm{s}_{\phi}^{2}} \\
& v_{3}=u_{2}(\beta-\alpha) \frac{\mathrm{s}_{\phi} \mathrm{c}_{\phi} \mathrm{c}_{\psi}^{2}}{\mathrm{c}_{\phi}^{2}\left(\alpha \mathrm{s}_{\psi}^{2}+\beta \mathrm{c}_{\psi}^{2}\right)+\alpha \mathrm{s}_{\phi}^{2}}
\end{aligned}
$$

The minimum then reads

$$
\begin{aligned}
\inf _{w_{1}, w_{3}} H\left(w_{1}, u_{2}, w_{3}\right) & =H\left(v_{1}, u_{2}, v_{3}\right) \\
& =\alpha \beta \frac{u_{2}^{2}}{\left(\mathrm{c}_{\phi}^{2}\left(\alpha \mathrm{s}_{\psi}^{2}+\beta \mathrm{c}_{\psi}^{2}\right)+\alpha \mathrm{s}_{\phi}^{2}\right)^{2}}\left(\beta \mathrm{c}_{\phi}^{2} \mathrm{c}_{\psi}^{2}+\alpha \mathrm{s}_{\psi}^{2} \mathrm{c}_{\phi}^{2}+\alpha \mathrm{s}_{\phi}^{2}\right) \\
& =u_{2}^{2} \frac{\alpha \beta}{\mathrm{c}_{\phi}^{2}\left(\alpha \mathrm{s}_{\psi}^{2}+\beta \mathrm{c}_{\psi}^{2}\right)+\alpha \mathrm{s}_{\phi}^{2}} .
\end{aligned}
$$

Case 3. Case 3 is the same as Case 2 up to switching $c_{\phi}$ and $\mathrm{s}_{\phi}$. Thus

$$
\inf _{w_{1}, w_{2}} H\left(w_{1}, w_{2}, u_{3}\right)=u_{3}^{2} \frac{\alpha \beta}{\mathrm{s}_{\phi}^{2}\left(\alpha \mathrm{s}_{\psi}^{2}+\beta \mathrm{s}_{\psi}^{2}\right)+\alpha \mathrm{c}_{\phi}^{2}}
$$


Slicing argument and convexity inequalities. In the three-dimensional case, the slicing argument and inequality (4.5) imply, with obvious notation,

$$
\begin{aligned}
\lambda_{2}= & \int_{(0,1)^{2}}\left(\alpha \int_{E_{1}^{2}\left(y_{1}, y_{3}\right)}|\nabla u|^{2} d y_{2}+\beta \int_{(0,1)^{2} \backslash E_{2}^{2}\left(y_{1}, y_{3}\right)}|\nabla u|^{2} d y_{2}\right. \\
& +\int_{\left[E_{2}^{2}\left(y_{1}, y_{3}\right) \backslash E_{1}^{2}\left(y_{1}, y_{3}\right)\right] \backslash E_{3}^{2}\left(y_{1}, y_{3}\right)}\left\langle\left(\begin{array}{ccc}
\beta & 0 & 0 \\
0 & \alpha & 0 \\
0 & 0 & \alpha
\end{array}\right) R(y) \nabla u, R(y) \nabla u\right\rangle d y_{2} \\
& \left.+\int_{E_{3}^{2}\left(y_{1}, y_{3}\right)}\left\langle\left(\begin{array}{ccc}
\alpha & 0 & 0 \\
0 & \beta & 0 \\
0 & 0 & \beta
\end{array}\right) R(y) \nabla u, R(y) \nabla u\right\rangle d y_{2}\right) d y_{1} d y_{3} \\
\geq & \int_{(0,1)^{2}} \inf \left\{\alpha \int_{E_{1}^{2}\left(y_{1}, y_{3}\right)} v^{\prime}\left(y_{2}\right)^{2} d y_{2}+\beta \int_{(0,1)^{2} \backslash E_{2}^{2}\left(y_{1}, y_{3}\right)} v^{\prime}\left(y_{2}\right)^{2} d y_{2}\right. \\
& +\int_{\left[E_{2}^{2}\left(y_{1}, y_{3}\right) \backslash E_{1}^{2}\left(y_{1}, y_{3}\right)\right] \backslash E_{3}^{2}\left(y_{1}, y_{3}\right)} \frac{v^{\prime}\left(y_{2}\right)^{2} \alpha \beta}{\mathrm{c}_{\phi}^{2}\left(\beta \mathrm{s}_{\psi}^{2}+\alpha \mathrm{c}_{\psi}^{2}\right)+\beta \mathrm{s}_{\phi}^{2}} d y_{2} \\
& \left.+\int_{E_{3}^{2}\left(y_{1}, y_{3}\right)} \frac{v^{\prime}\left(y_{2}\right)^{2} \alpha \beta}{\mathrm{c}_{\phi}^{2}\left(\alpha \mathrm{s}_{\psi}^{2}+\beta \mathrm{c}_{\psi}^{2}\right)+\alpha \mathrm{s}_{\phi}^{2}} d y_{2}, v \in H_{0}^{1}((0,1))\right\} d y_{1} d y_{3}
\end{aligned}
$$

Similar bounds can be derived for $\lambda_{1}$ and $\lambda_{3}$.

Next, we can use Proposition 2.5. In addition, Proposition 2.6 also holds in any dimension. Therefore

$$
\begin{aligned}
\frac{1}{\theta_{1}}+\frac{1}{\theta_{2}}+\frac{1}{\theta_{3}} \leq & 3 \theta_{1} \frac{1}{\alpha}+3\left(1-\theta_{2}\right) \frac{1}{\beta} \\
& +\int_{E_{3}}\left(\frac{\alpha \mathrm{c}_{\psi}^{2}+\beta \mathrm{s}_{\psi}^{2}}{\alpha \beta}+\frac{\mathrm{c}_{\phi}^{2}\left(\alpha \mathrm{s}_{\psi}^{2}+\beta \mathrm{c}_{\psi}^{2}\right)+\alpha \mathrm{s}_{\phi}^{2}}{\alpha \beta}\right. \\
& \left.+\frac{\mathrm{s}_{\phi}^{2}\left(\alpha \mathrm{s}_{\psi}^{2}+\beta \mathrm{s}_{\psi}^{2}\right)+\alpha \mathrm{c}_{\phi}^{2}}{\alpha \beta}\right) d y \\
& +\int_{\left(E_{2} \backslash E_{1}\right) \backslash E_{3}}\left(\frac{\beta \mathrm{c}_{\psi}^{2}+\alpha \mathrm{s}_{\psi}^{2}}{\alpha \beta}+\frac{\mathrm{c}_{\phi}^{2}\left(\beta \mathrm{s}_{\psi}^{2}+\alpha \mathrm{c}_{\psi}^{2}\right)+\beta \mathrm{s}_{\phi}^{2}}{\alpha \beta}\right) d y \\
= & \left.3 \theta_{1} \frac{1}{\alpha}+3\left(1-\theta_{2}\right) \frac{1}{\beta}+\theta_{3}\left(\frac{1}{\alpha}+\frac{2}{\beta}\right)+\left(\theta_{2}^{2}-\alpha \mathrm{s}_{\psi}^{2}\right)+\beta \mathrm{c}_{\phi}^{2}-\theta_{3}\right)\left(\frac{1}{\beta}+\frac{2}{\alpha}\right) \\
= & 3\left(\frac{\theta}{\alpha}+\frac{1-\theta}{\beta}\right),
\end{aligned}
$$

which is the desired harmonic bound.

4.3. Optimality of the bounds. Using the natural extensions of the twodimensional test functions of Section 3 to three dimensions, one can show the harmonic and arithmetic bounds to be attained. The entire convex set is also attained by layering. 
5. Interpretation in terms of $G$-closure. In this last section, we briefly recall a more classic way to derive the arithmetic and harmonic bounds, also known as the Voigt and Reuss bounds. We then interpret our results in terms of quasiconvex envelope and attainment of infima following the seminal papers of Kohn and Strang [9], based on the vectorization of the problem by Tartar [12, 13], and further studied by Allaire and Francfort [2].

5.1. Derivation of the trivial bounds. Let $n \in \mathbb{N}, \xi \in \mathcal{M}_{n}(\mathbb{R})$ and consider

$$
H_{0}^{1}\left((0,1)^{n}, \mathbb{R}^{n}\right) \ni u \mapsto F(\xi, \nabla u)=\int_{(0,1)^{n}}\langle A(y)(\xi+\nabla u), \xi+\nabla u\rangle d y
$$

The arithmetic bound is a consequence of

$$
\inf \left\{F(\xi, \nabla u): u \in H_{0}^{1}\left((0,1)^{n}\right)\right\} \leq F(\xi, 0)
$$

for $\xi=\left(\begin{array}{ll}1 & 0 \\ 0 & 1\end{array}\right)$. The derivation of the harmonic bound is related to the same minimization on an enlarged space, namely on

$$
L_{\#}^{2}\left((0,1)^{n}\right)^{n \times n}=\left\{v \in L^{2}\left((0,1)^{n}\right)^{n \times n}, \int_{(0,1)^{n}} v d y=0\right\} .
$$

In this case, a direct computation shows

$$
\begin{aligned}
\inf \left\{F(\xi, \nabla u): u \in H_{0}^{1}\left((0,1)^{n}\right\}\right. & \geq \inf \left\{F(\xi, v): v \in L_{\#}^{2}\left((0,1)^{n}\right)^{n \times n}\right\} \\
& =\left\langle\left(\int_{(0,1)^{n}} A^{-1}\right)^{-1} \xi, \xi\right\rangle .
\end{aligned}
$$

This inequality has an interpretation in terms of quadratic forms since the left-hand side is a quadratic form (quadratic forms are closed by $\Gamma$-convergence). Taking the inverse on both sides and reversing the inequality yields the harmonic bound for $\xi=\left(\begin{array}{ll}1 & 0 \\ 0 & 1\end{array}\right)$

This way of deriving the trivial bounds is more straightforward and holds for any dimension. However it does not immediately suggest the form of optimal configurations, as opposed to the present 'pointwise' approach.

5.2. Interpretation in terms of quasiconvexification. Following the approach initiated in [9], we may look for the harmonic bound as the minimum of a functional depending on the geometry of the different phases of the material in $(0,1)^{n}$.

Let $\chi_{E_{1}}$ and $\chi_{E_{2}}$ be the characteristic functions associated to the two isotropic phases $A_{1}=\left(\begin{array}{cc}\alpha & 0 \\ 0 & \alpha\end{array}\right)$ and $A_{2}=\left(\begin{array}{cc}\beta & 0 \\ 0 & \beta\end{array}\right)$. And let $A_{3}$ be the anisotropic phase. For $\xi \in \mathcal{M}_{n}(\mathbb{R})$, the objective is to minimize the functional

$$
J\left(\chi_{1}, \chi_{2}\right)=\int_{(0,1)^{n}}\left\langle\left(\chi_{1} A_{1}+\chi_{2} A_{2}+\left(1-\chi_{1}-\chi_{2}\right) A_{3}\right)\left(\xi+\nabla u_{\chi_{1}, \chi_{2}}\right), \xi+\nabla u_{\chi_{1}, \chi_{2}}\right\rangle d y
$$

on the space of admissible characteristic functions

$$
\begin{gathered}
\Xi=\left\{\left(\chi_{1}, \chi_{2}\right) \in L^{\infty}\left((0,1)^{n} ;\{0,1\}^{2}\right): \chi_{1} \chi_{2}=0, \int_{(0,1)^{n}}\left(\chi_{1}+\frac{1}{2}\left(1-\chi_{1}-\chi_{2}\right)\right) d y=\theta,\right. \\
\left.\int_{(0,1)^{n}}\left(\chi_{2}+\frac{1}{2}\left(1-\chi_{1}-\chi_{2}\right)\right) d y=1-\theta\right\},
\end{gathered}
$$


$\theta \in(0,1)$ being fixed. Here $u_{\chi_{1}, \chi_{2}}$ realizes the minimum of $F(\xi, \nabla(\cdot))$ on $H_{0}^{1}\left((0,1)^{n}\right)$, with $A(y)=\chi_{1} A_{1}+\chi_{2} A_{2}+\left(1-\chi_{1}-\chi_{2}\right) A_{3}$.

We then have

$$
\begin{aligned}
\inf _{\Xi} J\left(\chi_{1}, \chi_{2}\right)= & \inf _{\Xi} \inf _{u \in H_{\xi}} \int_{(0,1)^{n}}\left\langle\left(\chi_{1} A_{1}+\chi_{2} A_{2}+\left(1-\chi_{1}-\chi_{2}\right) A_{3}\right) \nabla u, \nabla u\right\rangle d y \\
= & \inf _{u \in H_{\xi}} \int_{(0,1)^{n} \chi_{1}, \chi_{2}=0,1 ; \chi_{1} \chi_{2}=0}\left[\left\langle\left(\chi_{1} A_{1}+\chi_{2} A_{2}\right.\right.\right. \\
& \left.\left.+\left(1-\chi_{1}-\chi_{2}\right) A_{3}\right) \nabla u, \nabla u\right\rangle+l_{1}\left(\chi_{1}+\frac{1}{2}\left(1-\chi_{1}-\chi_{2}\right)\right) \\
& \left.+l_{2}\left(\chi_{2}+\frac{1}{2}\left(1-\chi_{1}-\chi_{2}\right)\right)\right] d y
\end{aligned}
$$

where $H_{\xi}=\left\{u \in H^{1}\left((0,1)^{n}, \mathbb{R}^{n}\right): u(x)=\xi \cdot x\right.$ on $\left.\partial(0,1)^{n}\right\}$ and $l_{1}, l_{2}$ are Lagrange multipliers to impose the volume averages of $\chi_{1}$ and $\chi_{2}$.

Therefore, one can interpret the computation of the optimal bounds on the trace of the homogenized matrix as the computation of an infimum of a vector-valued integral functional. In order to apply the direct method of the calculus of variations, one may first compute the quasiconvex envelope of the integrand. The computation of a quasiconvex envelope is rarely explicit and this is the main reason why it may seems surprising at a first glance that we achieve this difficult task with such very simple arguments.

Actually, the bounds we have obtained being the trivial bounds, the quasiconvex envelope coincides with the convex envelope and

$$
\begin{aligned}
\inf _{\Xi} J\left(\chi_{1}, \chi_{2}\right)= & \inf _{v \in V_{\xi}} \int_{(0,1)^{n} \chi_{1}, \chi_{2}=0,1 ; \chi_{1} \chi_{2}=0}\left[\left\langle\left(\chi_{1} A_{1}+\chi_{2} A_{2}+\left(1-\chi_{1}-\chi_{2}\right) A_{3}\right) v, v\right\rangle\right. \\
& \left.+l_{1}\left(\chi_{1}+\frac{1}{2}\left(1-\chi_{1}-\chi_{2}\right)\right)+l_{2}\left(\chi_{2}+\frac{1}{2}\left(1-\chi_{1}-\chi_{2}\right)\right)\right] d y
\end{aligned}
$$

where $V_{\xi}=\left\{v \in L_{\#}^{2}\left((0,1)^{n \times n}: \int_{(0,1)^{n}} v=\xi\right\}\right.$. There exists a family of matrix fields for which this infimum is attained and which saturate the harmonic bound. The associated minimizers $v \in V_{\xi}$ turn out to belong to $H_{\xi}$. The matrices are represented on Figure 3.2 with varying $\theta_{1}$ and $\theta_{2}$ such that $\theta_{1}+\theta_{2}=2 \theta$.

Turning now to the arithmetic bounds, there also exists a family of matrix fields that saturate this inequality. These matrices are represented on Figure 3.1, with varying $\theta_{1}$ and $\theta_{2}$ such that $\theta_{1}+\theta_{2}=2 \theta$. In particular, they are divergence free.

5.3. Extensions to any number of conductivities and dimensions. The interpretation in terms of quasiconvexification and the generality of the Voigt and Reuss bounds allow us to state a general version of Theorem 1.

ThEOREM 3. Let $\left\{\alpha_{1}, \ldots, \alpha_{N}\right\}$ be $N$ ordered conductivities. We denote by $\mathcal{A}=\left\{A_{\beta_{1}, \ldots, \beta_{n}}\right\}$ the set of diagonal matrices of order $n$ such that $A_{\beta_{1}, \ldots, \beta_{n}}(i, i)=$ $\alpha_{\beta_{i}}$. Let $A:(0,1)^{n} \rightarrow \mathbb{R}^{n}$ be such that, up to a rotation and for almost all $x \in$ $(0,1)^{n}, A(x) \in \mathcal{A}$, we set $A=\sum_{\beta_{1}, \ldots, \beta_{n}=1, N} \chi_{\beta_{1}, \ldots, \beta_{n}} R(x)^{T} A_{\beta_{1}, \ldots, \beta_{n}} R(x)$, where $\chi_{\beta_{1}, \ldots, \beta_{n}}$ are characteristic functions and $R(x)$ are rotations. We denote by $\theta_{\beta_{1}, \ldots, \beta_{n}}=$ $\int_{(0,1)^{n}} \chi_{\beta_{1}, \ldots, \beta_{n}}$. Let now fix to $\theta_{\alpha_{i}} \in(0,1)$ the proportions of the conductivities $\alpha_{i}$. 
We have $\sum_{i=1, N} \theta_{\alpha_{i}}=1$. Consider the following relationships between the phases,

$$
\left\{\begin{array}{l}
\theta_{1, \ldots, 1}+\sum_{\beta_{n} \neq 1} \frac{1}{n} \theta_{1, \ldots, 1, \beta_{n}}+\cdots+\sum_{\beta_{2}, \ldots, \beta_{n} \neq 1} \frac{n-1}{n} \theta_{1, \beta_{2}, \ldots, \beta_{n}}=\theta_{\alpha_{1}} \\
\ldots \\
\theta_{N, \ldots, N}+\sum_{\beta_{n} \neq N} \frac{1}{n} \theta_{N, \ldots, N, \beta_{n}}+\cdots+\sum_{\beta_{2}, \ldots, \beta_{n} \neq N} \frac{n-1}{n} \theta_{N, \beta_{2}, \ldots, \beta_{n}}=\theta_{\alpha_{N}}
\end{array},\right.
$$

the associated $G$-closure is the bounded set of $\lambda_{1}, \ldots, \lambda_{n}$ satisfying simultaneously all the following inequalities in eigenvalue space

$$
\left\{\begin{array}{l}
\alpha_{1} \leq \lambda_{i} \leq \alpha_{N} \\
\sum_{i=1, n} \lambda_{i} \leq n \sum_{j=1, N} \theta_{\alpha_{j}} \alpha_{j} \\
\sum_{i=1, n} \frac{1}{\lambda_{i}} \leq n \sum_{j=1, N} \theta_{\alpha_{j}} \frac{1}{\alpha_{j}}
\end{array}\right.
$$

An example of optimal configuration is given for the arithmetic bounds in Figure 5.1. The rotation of $\pi / 2$ of this configuration gives an optimal configuration for the harmonic bound.

\begin{tabular}{|l|l|l|l|}
\hline 41 & 42 & 43 & 44 \\
\hline 31 & 32 & 33 & 34 \\
\hline 21 & 22 & 23 & 24 \\
\hline 11 & 12 & 13 & 14 \\
\hline
\end{tabular}

FIG. 5.1. Optimal geometry for the arithmetic bound in 2 dimensions for 4 conductivities

It should be emphasized that these rank-one laminate configurations correspond to the general class introduced in $[8,15]$ for multiphase materials.

The exact bounds are plotted on Figure 5.2 for a three layer-type composite $\alpha=1$, $\beta=2, \gamma=3$ with the proportions $\theta_{\alpha}=\theta_{\beta}=\theta_{\gamma}=1 / 3$.

Acknowledgements. We thank Enzo Nesi for valuable comments and help concerning the link of the present work with the theory of $G$-closure, and acknowledge support from the Marie Curie Research Training Network MRTN-CT-2004-505226 'Multi-scale modelling and characterisation for phase transformations in advanced materials' (MULTIMAT).

\section{REFERENCES}

[1] R. Alicandro and M. Cicalese. Representation result for continuum limits of discrete energies with superlinear growth. SIAM J. Math Anal. 36 (2004), 1-37

[2] G. Allaire and G. Francfort. Existence of minimizers for non-quasiconvex functionals arising in optimal design, Ann. Inst. H. Poincarè Anal. Non Linèaire, 15 (1998), 301-339.

[3] A. Braides. Г-convergence for Beginners, Oxford University Press. Oxford, 2002.

[4] A. Braides. A handbook of $\Gamma$-convergence. In Handbook of Differential Equations, Stationary Partial Differential Equations, Vol. 3 (M. Chipot and P. Quittner eds.), Elsevier, 2006. 


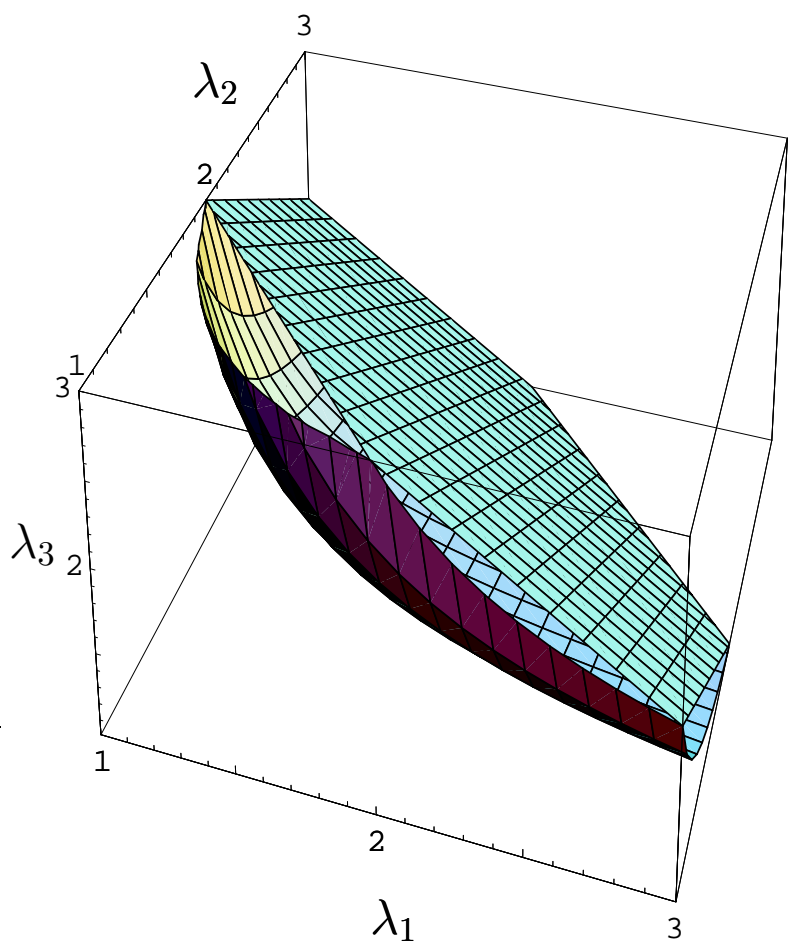

FIG. 5.2. Exact bounds in 3 dimensions (note that $1 \leq \lambda_{i} \leq 3$ )

[5] A. Braides and A. Defranceschi. Homogenization of Multiple Integrals. Oxford University Press, Oxford, 1998.

[6] A.Braides and G.A. Francfort. Bounds on the effective behaviour of a square conducting lattice, Proc. R. Soc. Lond. A (2004) 460, 1755-1769.

[7] G. Dal Maso. An Introduction to $\Gamma$-convergence, Birkhäuser, Boston, 1993.

[8] L.V. Gibiansky and O. Sigmund. Multiphase composites with extremal bulk modulus, J. Mech. Phys. Solids 48 (2000), 461-498.

[9] R.V. Kohn and G. Strang. Optimal design and relaxation of variational problems, I, II and III, Comm. Pure Appl. Math. 39 (1986).

[10] K. Lurie and A.V. Cherkaev. Exact estimates of conductivity of mixtures composed of two materials taken in prescribed proportion (plane problem). Doklady Akademii Nauk SSSR 264 (1982), 1129-1130.

[11] G.W. Milton. The Theory of Composites. Cambridge Monographs on Applied and Computational Mathematics, 6, Cambridge University Press, Cambridge, 2002.

[12] F. Murat and L. Tartar. H-convergence. In: Topics in the mathematical modelling of composite materials, A.V. Cherkaev and R.V. Kohn, eds.. Progress in nonlinear differential equations and their applications, 31, Birkhaüser, Boston, 1997, 21-44.

[13] F. Murat. H-Convergence, Séminaire d'Analyse Fonctionnelle et Numérique, Univ. Alger, Multigraphié, 1978.

[14] U. Raitums. On the local representation of G-closure. Arch. Ration. Mech. Anal. 158 (2001), 213-234.

[15] O. Sigmund. A new class of extremal composites. J. Mech. Phys. Solids 48 (2000), 397-428.

[16] L. Tartar. Estimation de coefficients homogénéisés. In: Computing methods in applied sciences and engineering (Proc. Third Internat. Sympos., Versailles, 1977), I, pp. 364-373, Lecture Notes in Math., 704, Springer, Berlin, 1979.

[17] L. Tartar. Estimations fines de coefficients homogénéisés. In: Ennio De Giorgi Colloquium, P. Krée, ed. Pitman, Reseach Notes in Mathematics 125, London, 1985, 168-187. 